(C) 2019 IEEE. Personal use of this material is permitted. Permission from IEEE must be obtained for all other uses, in any current or future media, including reprinting/republishing this material for advertising or promotional purposes, creating new collective works, for resale or redistribution to servers or lists, or reuse of any copyrighted component of this work in other works.

\title{
BATCH FABRICATION OF MULTILAYER POLYMER CANTILEVERS WITH INTEGRATED HARD TIPS FOR HIGH-SPEED ATOMIC FORCE MICROSCOPY
}

\author{
Nahid Hosseini ${ }^{l}$, Oliver Peric ${ }^{1}$, Matthias Neuenschwander ${ }^{l}$, Santiago H. Andany ${ }^{l}$, \\ Jonathan D. Adams ${ }^{2}$, and Georg E. Fantner ${ }^{1}$ \\ ${ }^{1}$ Laboratory for Bio- and Nano- Instrumentation, Ecole Polytechnique Fédérale de \\ Lausanne, Lausanne, Switzerland and \\ ${ }^{2}$ Biophysik department, ETH Zürich, Basel, Switzerland
}

\begin{abstract}
Increasing the speed of AFM imaging has significant benefits for academic research as well as industrial applications. In many imaging modes, the dynamic response of the cantilever probe dictates the achievable speed. Polymer cantilevers have gained great attention due to their high tracking ability and ease of fabrication. However, polymer cantilevers also have drawbacks. Polymers are not well suitable materials for the tip of the probe due to their high wear rate. This has limited the broader use of polymer cantilevers for AFM imaging. In this work, we combine the advantages of polymer cantilevers with the advantages of cantilevers made of conventional MEMS materials. We demonstrate the batch integration of a hard tip into a polymer-core multilayer cantilever probe, thereby merging speed, high-resolution and durability in a single cantilever.
\end{abstract}

\section{INTRODUCTION}

Cantilever based sensing methods, such as atomic force microscopy (AFM) and related techniques, have become one of the main ways to probe materials in the nanoscale regime [1]. Mechanical properties of the cantilevers influence AFM imaging speed (mechanical bandwidth), image resolution (tip radius) and the analysis of specific samples (cantilever stiffness). The mechanical bandwidth of a cantilever operated in AC mode can be estimated through the ratio $f_{0} / Q$, where $f_{0}$ is the first resonance frequency and $\mathrm{Q}$ is the quality factor of the cantilever. Therefore, an increase of the resonance frequency or a decrease of the quality factor will result in higher mechanical bandwidth. Current high-speed AFM (HS-AFM) technology was enabled by the miniaturization of silicon and silicon nitride ( $\mathrm{SiN}$ ) cantilevers to planar dimensions below 10 microns, resulting in cantilevers with resonance frequencies in the $\mathrm{MHz}$ range [2], [3]. This approach has been the enabling technology for state of the art, high-speed AFM, producing good-quality, high-speed images even on difficult biological samples. In liquid, viscous damping yields a $Q$ value around 2-4, enabling extremely high imaging speeds. Using small cantilevers immersed in fluid, Ando et al. [3] pioneered video-rate AFM imaging and thereby established the field of HSAFM in liquid on biological samples. Although such cantilevers may also have a relatively low Q in air, the small spring constants make them primarily intended for imaging in fluid. In air, higher spring constants are often needed to overcome surface adhesion.

We have recently shown that making probes out of polymers yields very fast-responding cantilevers, and polymers are thus excellent structural materials for highspeed AFM probes [4]. By changing the material to a polymer, we can mimic the hydrodynamic damping effect that occurs in liquid, thus obtaining a low quality-factor independent of the medium. Pioneering work by Genolet et al [5] has shown that cantilevers with integrated tips can be made out of the polymer SU8 using a silicon mold to form the cantilever tip. These tips can have acceptable radii for various imaging purposes. However, the wear rate of SU8 is very high [6] which makes SU8 and other polymers nonideal for serving as tip material. Previous attempts for making cantilevers as well as the cantilever carrying chip out of polymer, has shown that mechanical excitation of the cantilever resonance is difficult, and the cantilevers do not show a clean tune required for good tapping mode AFM operation. In this work, we present an alternative strategy to integrate sharp hard tips with acceptable durability into polymer cantilevers. By using non-traditional MEMS materials in combination with traditional materials and fabrication methods, we have fabricated hybrid polymer AFM cantilevers made of a polymer core, sandwiched between two hard thin films. The carrier chip of the cantilever is made of silicon, which facilitates easy excitation of the cantilever resonance when using inertial drive. The reported tri-layer cantilevers maintain high tracking bandwidth, governed by the viscoelastic properties of the polymer core, combined with improved tip durability.

\section{FABRICATION}

Our approach to fabricate high-speed, hard tip, polymer core cantilevers is to first fabricate the sharp hardtips on the wafer, which we then bond to a complementary wafer through polymer bonding. The bonded wafers are then further processed to obtain the final cantilever. The main steps of the process flow are depicted in Figure 1.

We use a standard four-inch, double-sided polished, silicon (Si) wafer with a thickness of $380 \mu \mathrm{m}$ (hereafter called wafer 1). The first step is to deposit a 20-100nm thin layer of low stress silicon nitride (SiN) through LPCVD. Then, we use e-beam lithography to write the circular pattern and RIE to transfer the pattern onto the SiN layer. The circular patterns for the openings provide maximum symmetry, which, after $\mathrm{KOH}$ wet etching, results in pyramidal shaped pits. The SiN mask is then removed in diluted HF. We use thermal oxidation to transform silicon layer into silicon oxide $\left(\mathrm{SiO}_{2}\right)$, which results in a sharpening of the mold [7]. Next, we deposit low stress SiN through LPCVD. The deposited SiN layer will constitute the outermost layer of the tri-layer cantilever and its 
thickness can vary from $20 \mathrm{~nm}$ to $100 \mathrm{~nm}$. A complementary double-sided polished silicon wafer (wafer 2) with the same SiN layer thickness is bonded onto wafer 1 (Figure 1$\mathrm{v})$. The bonding material is a viscoelastic polymer responsible for the low quality-factor of the cantilever. We use benzocyclobutene (BCB), with the commercial product named CYCLOTENE 3022 (The Dow Chemical Company) for wafer bonding. $\mathrm{BCB}$ is suitable for a polymer layer thickness of $2-11 \mu \mathrm{m}$ and can be spin coated on one or both wafers before bonding. For wafer bonding, we use an SB6 bonding machine (SüssMicrotech). The bonded wafers are then hard cured under nitrogen atmosphere at $250^{\circ} \mathrm{C}$ for 60 minutes. (a)

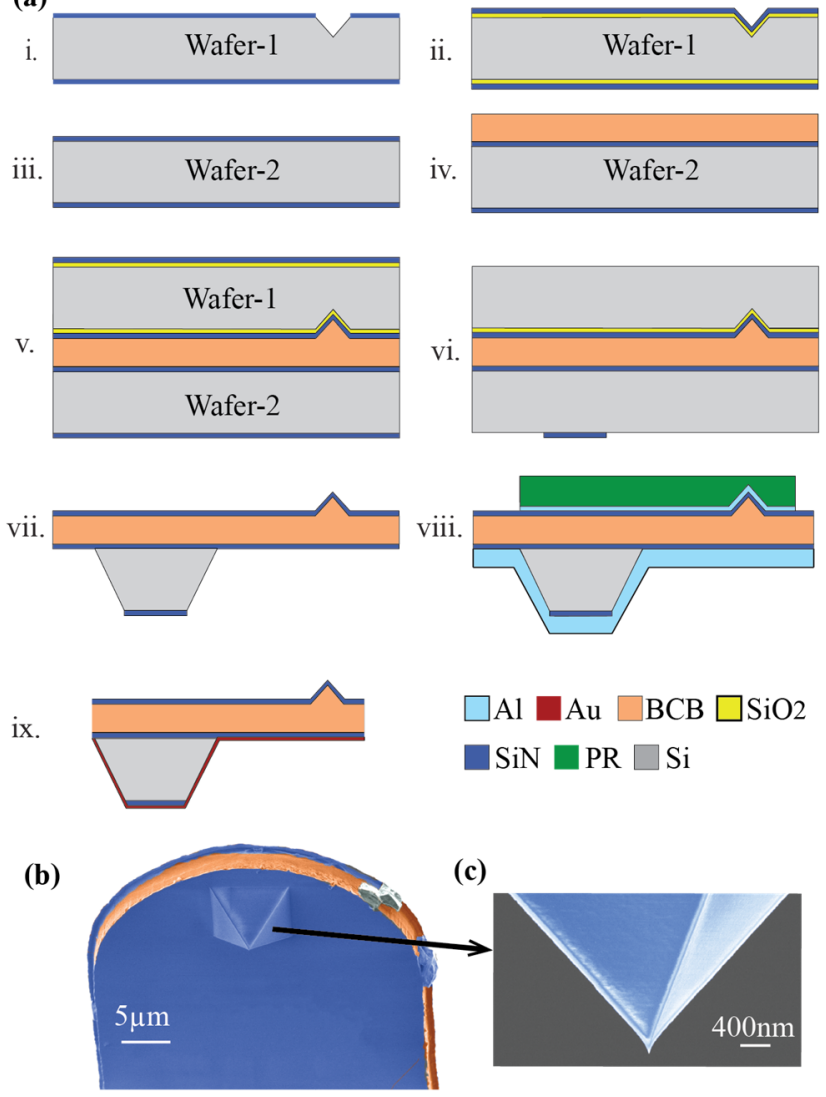

Figure 1: Process flow, displaying the most important steps. (a) The process is based on polymer bonding of two pre-processed wafers 1\&2. (b), (c) SEM images of a released cantilever and its tip. The $B C B$ polymer core (in orange), is sandwiched between two hard SiN thin films of identical thicknesses (in blue). The hard tip is made of SiN.

Continuing the process flow, we pattern wafer 1 by standard photolithography and dry etching. On wafer 2, we strip the $\mathrm{SiO}_{2}$ and $\mathrm{SiN}$ layers, thus exposing the silicon. Thereafter we etch the wafer assembly in $40 \% \mathrm{KOH}$ at $60^{\circ} \mathrm{C}$ overnight with a total etch time of $\sim 19 \mathrm{~h}$ (Figure 1 vii). As the $\mathrm{BCB}$ layer is covered on both sides with a $\mathrm{SiN}$ layer, it resists the hours-long etch. The thermally grown oxide is attacked during the $\mathrm{KOH}$ etch and we later completely remove it in buffered HF. Subsequently, we deposit a $2 \mu \mathrm{m}$ thick aluminum (Al) layer on the chip body side (wafer 2), which serves as a mechanical support layer for the thin SiN-BCB-SiN membrane. On the cantilever tip side, we deposit a $300 \mathrm{~nm}$ thin aluminum layer, which acts as a hard mask during consecutive dry etching. To pattern the aluminum on the cantilever tip side of the wafer, we use a $12 \mu \mathrm{m}$ thick photoresist (PR) (AZ 9260, MicroChemicals) in order to completely cover the cantilever tips. The patterned aluminum is wet etched (Figure 1-viii).

The thick aluminum layer on the chip body side of the wafer is necessary as a structural layer, since the final BCB dry etch defines the cantilever shape and removes the surrounding BCB layer. The BCB dry etch consists of three main etching steps, where the first and the last etch step consist in removing the $\mathrm{SiN}$ layer using $\mathrm{CHF}_{3} / \mathrm{SF}_{6}$ chemistry. During the second etch step, we remove the $\mathrm{BCB}$ polymer layer using $\mathrm{CHF}_{3}$ chemistry. After $\mathrm{BCB}$ dry etching, we remove the aluminum layers on both sides using wet aluminum etchant. The final step is the deposition of a thin reflective layer on the chip body side of the wafer. We first sputter a $5 \mathrm{~nm}$ layer of titanium (adhesion layer) followed by 20nm of gold (Figure 1-ix). The cantilever with the integrated hard tip at the end of the process flow is depicted in Figure 1-b and the close-up of the tip is shown in Figure 1-c.

\section{RESULTS}

To evaluate the tip sharpness and wear resistance of the fabricated hard tips, the tri-layer cantilevers are tested by imaging a polycrystalline titanium roughness sample. The results are reported in Figure 2. This standard characterization sample for tip sharpness is well suited for tip evaluation as the peaks have steeper slopes than the reported cantilever tips. Tip sharpness is quantified using the blind tip estimation algorithm [8] of the Gwyddion software [9]. The algorithm identifies the sharpest peak in the topography image which is subsequently used to compute the tip sharpness at $10 \mathrm{~nm}$ from the apex, as illustrated in Figure 2-a. The images are obtained using a Bruker AFM system (Santa Barbara, CA, USA) comprising a Nanoscope-V controller, a MultiMode-V and a J-scanner. Images are taken in tapping mode and imaging parameters are as follows: $2 \times 2 \mu \mathrm{m}$ scan size, $1.95 \times 1.95 \mathrm{~nm}$ pixel size and $1 \mathrm{~Hz}$ scan rate. Measured tip radii range from $12 \mathrm{~nm}$ to $20 \mathrm{~nm}$ and are comparable to commercial cantilevers such as ScanAsyst-Fluid probes (Bruker), and are suitable for most AFM applications. The durability of the fabricated tips was tested during 11 hours of uninterrupted imaging, for a total of $170 \mathrm{~mm}$ of tip-travel distance. The 37 images obtained were analysed to detect potential tip degradation issues. Figures 2-b reveal no apparent degradation of the tip, even after prolonged imaging on a demanding sample.

Traditional polymer cantilevers with soft polymer chip bodies suffer from poor cantilever tunes. This issue is addressed here by using silicon chip bodies, drastically improving the mechanical response to excitation during inertial drive as illustrated in Figure 3. The tune of two cantilevers with similar first resonance frequencies are compared. The full SU8 lever (red curve) shows a more chaotic tune when compared to the reported tri-layer cantilever (blue curve). Extracting the correct first resonance of the cantilever is thus greatly facilitated by using a hard, silicon chip body. 
(a)

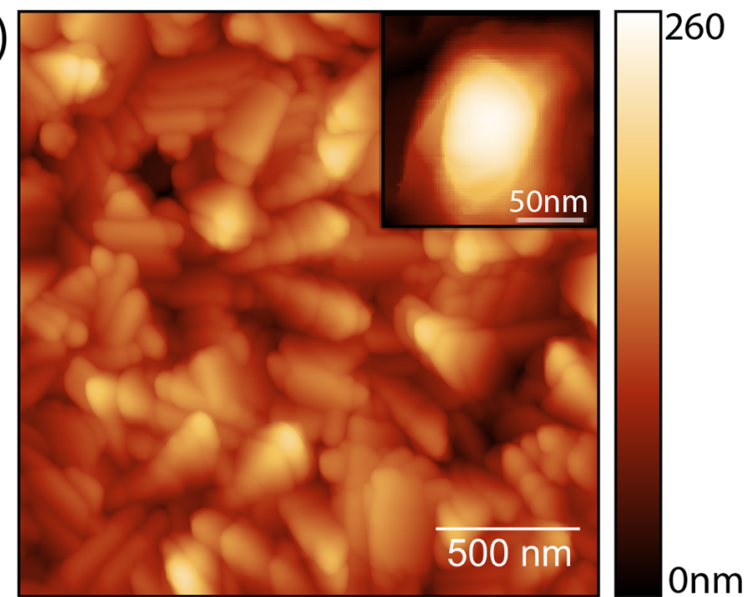

(b)

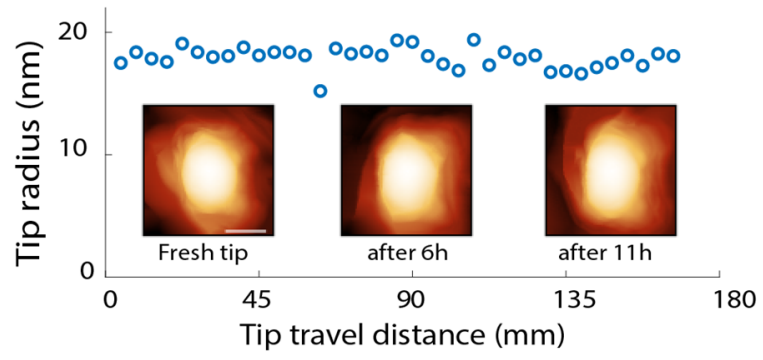

Figure 2: Tip sharpness and tip wear characterization through imaging of a titanium polycrystalline roughness sample. (a) Tapping mode image reveals a tip radius of $17 \mathrm{~nm}$, at 10nm from the apex. The Inset shows the region used for the Gwyddion blind tip estimation. (b) Evolution of tip sharpness as a function of imaging time and total tiptravel distance shows minimal tip wear, even after $11 \mathrm{~h}$ of imaging and $170 \mathrm{~mm}$ of tip travel distance.

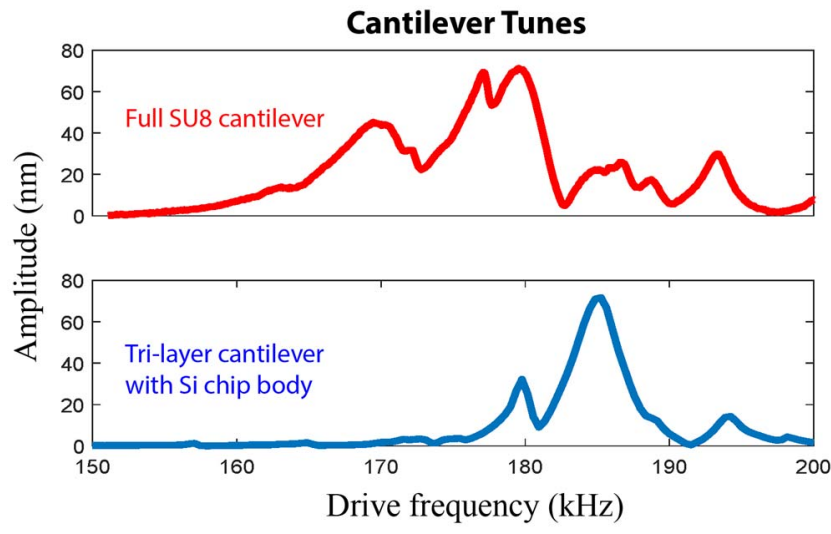

Figure 3: Cantilever tunes for an SU8 cantilever (red curve) and for a tri-layer cantilever with a silicon chip body (blue curve). Tri-layer lever characteristics: $Q=58, f_{0}=184 \mathrm{kHz}$, length $100 \mu \mathrm{m}$, width $50 \mu \mathrm{m}$ and thickness $4 \mu \mathrm{m}$ BCB core and 20nm LSNT shell. SU8 lever characteristic $Q=21, f_{0}=176 \mathrm{kHz}$, length $120 \mu \mathrm{m}$, width $30 \mu \mathrm{m}$ and thickness $8 \mu \mathrm{m}$.

To assess the imaging speed of the tri-layer cantilevers, we measured its detection bandwidth in tapping mode and compared it to a commercial silicon cantilever (RTESPA, Bruker AFM probes, Camarillo, CA, USA). To do so, we defined the tapping bandwidth as the $3 \mathrm{~dB}$ decrease in tracking amplitude and used a similar protocol than Kokavecz et al and Sulcheck et al [10]. The experiment (Figure 4-a) showed a more than 10 times higher bandwidth for the tri-layer cantilever $\left(\mathrm{f}_{0}=359 \mathrm{kHz}, \mathrm{k}=7.2 \mathrm{~N} / \mathrm{m}, \mathrm{Q}=\right.$ 55, planar dimensions $90 \mu \mathrm{m}$ by $30 \mu \mathrm{m}$ and thickness $2.6 \mu \mathrm{m})$ than for its silicon counterpart $\left(\mathrm{f}_{0}=339 \mathrm{kHz}, \mathrm{k}=\right.$ $48 \mathrm{~N} / \mathrm{m}, \mathrm{Q}=592$, planar dimension $125 \mu \mathrm{m}$ by $40 \mu \mathrm{m}$ and thickness $3.4 \mu \mathrm{m}$ ). In order to evaluate the correlation between tapping bandwidth and image quality at high speeds, we imaged an AFM calibration grating $(10 \mu \mathrm{m}$ pitch, 200nm depth) with the same two cantilevers, at $1 \mathrm{~Hz}$ and $10 \mathrm{~Hz}$ scan rate (Figure 4-b). While the silicon cantilever clearly tracks the sample poorly at $10 \mathrm{~Hz}$ scan rate, the tri-layer cantilever detects the sample topography significantly better.

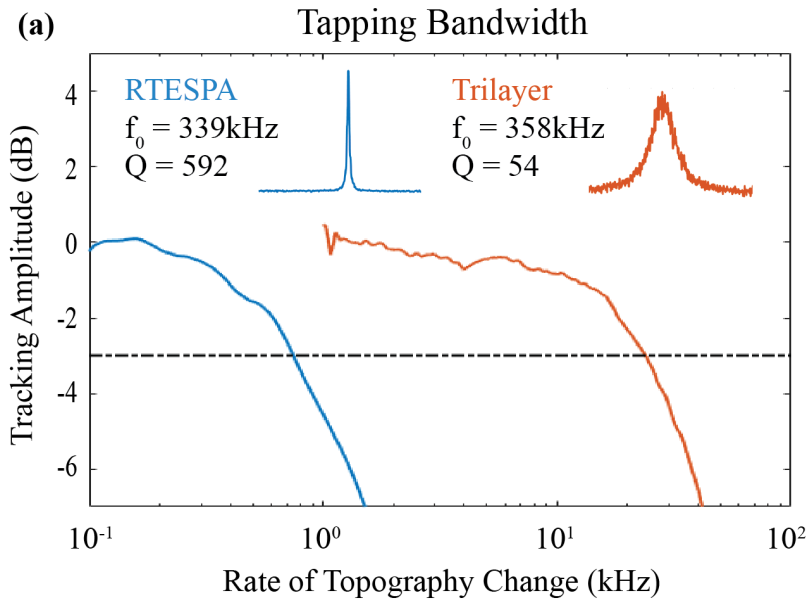

(b) Amplitude error

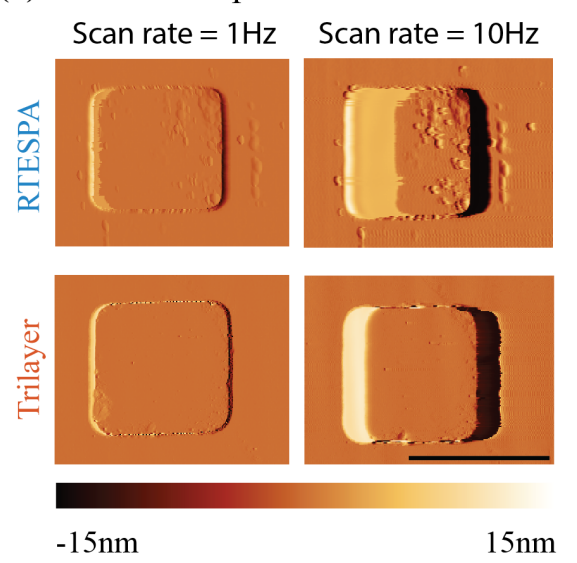

Figure 4: Comparison of tapping bandwidth between a trilayer cantilever and a commercial silicon cantilever (RTESPA). (a) The $3 d B$ drop of the surface tracking in tapping mode for the RTESPA and the tri-layer cantilever occurs at $750 \mathrm{~Hz}$ and $24 \mathrm{kHz}$, respectively. Both cantilevers have comparable resonance frequencies, but the tri-layer has a 10 times lower $Q$-factor. The insets show the thermal tune of each cantilever, at identical scale. (b) Amplitude error images of a $10 \mu \mathrm{m}$ pitch, $200 \mathrm{~nm}$ step reference sample taken by RTESPA and the tri-layer cantilever at $1 \mathrm{~Hz}$ and $10 \mathrm{~Hz}$ scan rates. The tri-layer cantilever shows better topography tracking ability thanks to its higher tapping bandwidth. The scale bar is $5 \mu \mathrm{m}$. 


\section{DISCUSSION}

The present work proposes a way to overcome the primary limitation of polymer AFM cantilevers, which is the poor wear rate of polymer tips and difficult mechanical excitation. By moving from the pure-polymer design to the tri-layer sandwich structure, one can benefit from the highspeed imaging capability of the polymer cantilevers and combine it with the use of tips that are made from the material that is known and accepted in the field as being suitable for high-quality tips. The tri-layer structure was chosen over a bilayer structure $(\mathrm{SiN}-\mathrm{BCB})$ to provide symmetry around the neutral axis and thus avoid cantilever bending due to internal stresses in the films. Compared to Si cantilevers, the tri-layer cantilevers have a 10 times higher imaging bandwidth. However, compared to pure polymer cantilevers (Adams et. al), the tri-layer cantilevers are slower. This is due to the over-proportional contribution of the $\mathrm{SiN}$ layer to the second moment of area of the cantilever, even for ultra-thin layers. The Young's modulus of the $\mathrm{SiN}$ (e.g. 240GPa) is two orders of magnitude higher compared to the BCB (e.g. 2.9GPa) layer, and the contribution of the $\mathrm{SiN}$ layer to the second moment of area depends on the distance to the neutral axis squared. It is therefore desirable to keep the SiN layer thickness as low as possible (20nm in our case). In the future we aim to replace $\mathrm{SiN}$ with materials with lower stiffness, for instance silicon oxide to obtain hard tip cantilevers with even lower Q-factor and hence even higher tracking speed.

An additional advantage of our proposed process is that all high temperature steps required for fabricating the tip are performed before bonding with the BCB polymer. This allows the integration of other functionalities to the tri-layer cantilevers by adapting the microfabrication recipe. For instance, by pre-patterning the $\mathrm{BCB}$ layer, microfluidic devices could be realized with the same technology with inherently sealed channels.

\section{CONCLUSION}

In this work, we have reported a technique to resolve the problem of high wear-rate and difficult mechanical excitation in polymer cantilevers by making sharp tips out of thin films with high in-plane stiffness and low out-ofplane stiffness. We have developed high-speed tri-layer AFM cantilevers where the polymer core, e.g. $\mathrm{BCB}$, is sandwiched between two $\mathrm{SiN}$ thin films through wafer bonding. The average tip radius for randomly selected cantilevers was measured to be $12 \mathrm{~nm}$ at a $10 \mathrm{~nm}$ distance from the tip apex. Long-term imaging showed negligible tip wear, even after $170 \mathrm{~mm}$ tip traveling distance during $11 \mathrm{~h}$ of uninterrupted imaging.

The good tip quality combines with the high tracking ability of the tri-layer for a versatile cantilever for AMAFM. The $3 \mathrm{~dB}$ decrease in tracking amplitude demonstrates more than one order of magnitude improvement in the tracking ability of the tri-layer cantilevers compared to its traditional silicon counterpart. We believe these crucial improvements remove the disadvantages of previous polymer-based cantilevers and tri-layer cantilevers suitable for use in routine AFM imaging applications.

\section{ACKNOWLEDGMENTS}

We thank the Center of Micronanotechnology (CMI) at EPFL for their help during microfabrication. This work was funded by the European Union FP7/2007-2013/ERC under Grant Agreement No. 307338-NaMic, the ERC2017-CoG; InCell; Project number 773091, and Swiss National Science Foundation (Nos.205321_134786, 205320_152675)

\section{REFERENCES}

[1] R. García, R. Magerle, and R. Perez, "Nanoscale compositional mapping with gentle forces," Nat. Mater., vol. 6, no. 6, pp. 405-411, Jun. 2007.

[2] D. A. Walters, J. P. Cleveland, N. H. Thomson, P. K. Hansma, M. A. Wendman, G. Gurley, and V. Elings, "Short cantilevers for atomic force microscopy," Rev. Sci. Instrum., vol. 67, no. 10, pp. 3583-3590, 1996.

[3] T. Ando, N. Kodera, E. Takai, D. Maruyama, K. Saito, and A. Toda, "A high-speed atomic force microscope for studying biological macromolecules," Proc. Natl. Acad. Sci., vol. 98, no. 22, pp. 12468-12472, 2001.

[4] J. D. Adams, B. W. Erickson, J. Grossenbacher, J. Brugger, A. Nievergelt, and G. E. Fantner, "Harnessing materials damping properties for high-speed atomic force microscopy," Nat. Nanotechnol., vol. in press, no. November, pp. 1$6,2015$.

[5] G. Genolet, J. Brugger, M. Despont, U. Drechsler, P. Vettiger, N. F. de Rooij, and D. Anselmetti, "Soft, entirely photoplastic probes for scanning force microscopy," Rev. Sci. Instrum., vol. 70, no. 5, pp. 2398-2401, May 1999.

[6] K. Sidler, "Fabrication and Characterization of SU8 Cantilevers with Integrated Tips Designed for Dip-Pen Nanolithography," p. Master's thesis, mechanical engineering, ETH, 2006.

[7] T. S. Ravi, R. B. Marcus, and D. Liu, "Oxidation sharpening of silicon tips," J. Vac. Sci. Technol. B Microelectron. Nanom. Struct., vol. 9, no. 6, p. 2733, Nov. 1991.

[8] J. S. Villarrubia, "Algorithms of Scanned Probe Microscope Image," J. Res. Natl. Inst. Stand. Technol., vol. 102, no. 4, pp. 425-454, 1997.

[9] D. Nečas and P. Klapetek, "Gwyddion: An opensource software for SPM data analysis," Cent. Eur. J. Phys., vol. 10, no. 1, pp. 181-188, 2012.

[10] T. Sulchek, G. G. Yaralioglu, C. F. Quate, and S. C. Minne, "Characterization and optimization of scan speed for tapping-mode atomic force microscopy," Rev. Sci. Instrum., vol. 73, no. 8, pp. 2928-2936, Aug. 2002. 